\title{
Positive Predictive Value of Neck Imaging Reporting and Data System Categories 3 and 4 Posttreatment FDG-PET/CT in Head and Neck Squamous Cell Carcinoma
}

\author{
(D) P. Wangaryattawanich, (D)B.F. Branstetter, (D).D. Ly, (D) U. Duvvuri, DD.E. Heron, and (DT.J. Rath
}

\begin{abstract}
BACKGROUND AND PURPOSE: The Neck Imaging Reporting and Data System is a standardized reporting system intended to risk stratify patients treated for head and neck squamous cell carcinoma. The purpose of this study is to investigate the positive predictive value of the Neck Imaging Reporting and Data System categories 3 and 4 on posttreatment PET/CT in patients treated definitively for head and neck squamous cell carcinoma.
\end{abstract}

MATERIALS AND METHODS: We retrospectively identified patients treated definitively for head and neck squamous cell carcinoma between 2006 and 2018. Patients whose posttreatment PET/CT scans were interpreted as Neck Imaging Reporting and Data System 3 (suspicious) or 4 (definitive recurrence) at the primary site, regional nodes, or at distant sites were included. The reference standard was histopathology or unequivocal imaging or clinical evidence of treatment failure. The positive predictive values of Neck Imaging Reporting and Data System 3 and 4 posttreatment PET/CT were calculated.

RESULTS: Seventy-two of 128 patients with posttreatment PET/CT interpreted as Neck Imaging Reporting and Data System 3 at the primary site, regional nodes, or distant sites were proved to have treatment failure at the suspicious sites, yielding an overall positive predictive value of $56 \%(95 \% \mathrm{Cl}, 48 \%-65 \%)$. The positive predictive values of Neck Imaging Reporting and Data System 3 by subsite were as follows: primary site, 56\% (44/79); regional nodes, 65\% (34/52); and distant sites, $79 \%$ (42/53). All 69 patients with posttreatment PET/CT interpreted as Neck Imaging Reporting and Data System 4 had true treatment failure, yielding a positive predictive value of $100 \%(95 \% \mathrm{Cl}, 96 \%-100 \%)$ : primary site, $100 \%$ (28/28); regional nodes, $100 \%$ (32/32); and distant sites, $100 \%(29 / 29)$.

CONCLUSIONS: The positive predictive value of Neck Imaging Reporting and Data System 3 on posttreatment PET/CT is relatively low. Thus, Neck Imaging Reporting and Data System 3 findings should be confirmed with tissue sampling before instituting new salvage treatment regimens to avoid unnecessary overtreatment and its associated toxicities. Neck Imaging Reporting and Data System 4 reliably indicates recurrent disease.

ABBREVIATIONS: AJCC = American Joint Committee on Cancer; $\mathrm{D}=$ distant sites; $\mathrm{HNSCC}=$ head and neck squamous cell carcinoma; HPV $=$ human papillomavirus; $\mathrm{N}=$ regional nodes; NI-RADS = Neck Imaging Reporting and Data System; $\mathrm{P}=$ primary site; PPV = positive predictive value

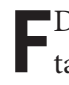
DG-PET/CT is a powerful imaging tool and critically important for management of patients with head and neck squamous cell carcinoma (HNSCC). Before treatment, PET/CT can be used for tumor staging, especially in patients with stage III and

Received November 29, 2019; accepted after revision April 4, 2020.

From the Departments of Radiology (P.W., B.F.B., J.D.L.), Otolaryngology (B.F.B., U.D., D.E.H.), Biomedical Informatics (B.F.B.), and Radiation Oncology (D.E.H.), School of Medicine, University of Pittsburgh, Pittsburgh, Pennsylvania; and Neuroradiology Section, Department of Radiology (T.J.R.), Mayo Clinic Hospital, Phoenix, Arizona.

Paper previously presented as an oral presentation at: Annual Meeting of the American Society of Neuroradiology, May 18-23, 2019: Boston, Massachusetts. Please address correspondence to Tanya J. Rath, MD, Neuroradiology Section, Department of Radiology, Mayo Clinic Arizona, 5777 E Mayo Blvd, Phoenix, AZ 8505; e-mail: Rath.Tanya@mayo.edu

http://dx.doi.org/10.3174/ajnr.A6589
IV disease, and also for localization of occult primary tumors. After treatment, PET/CT can be used for assessment of treatment response and disease surveillance. ${ }^{1-3}$ It has been well-established that posttreatment PET/CT has a high negative predictive value and patients who have complete tumor response on posttreatment PET/CT can avoid an unnecessary operation. ${ }^{4-6}$ The clinical impact of positive posttreatment PET/CT findings is less well-studied.

The Neck Imaging Reporting and Data System (NI-RADS) is a standardized report template used for surveillance contrastenhanced CT with or without concurrent PET. The primary objective of this template is to simplify radiology reports and facilitate communication between radiologists and their clinical colleagues. The results of posttreatment imaging surveillance are classified into 4 numeric categories based on the radiologist's 
suspicion for residual or recurrent tumor (category 1 , no evidence of recurrence; category 2, low suspicion; category 3 , high suspicion; category 4 , definitive disease recurrence). ${ }^{7-9}$ High negative predictive values of NI-RADS 1 and 2 on posttreatment PET/CT in HNSCC have been established. ${ }^{5,10}$ However, there are inadequate data regarding positive predictive values (PPVs) of NIRADS 3 and 4 as seen on posttreatment PET/CT.

The purpose of this study was to determine the PPV of NIRADS categories 3 and 4 on posttreatment PET/CT in patients treated definitively for HNSCC.

\section{MATERIALS AND METHODS \\ Study Design and Patient Selection}

We conducted a retrospective study that was approved by our institutional review board (PRO08120419) and was in compliance with the Health Insurance Portability and Accountability Act. Patient data were obtained from the University of Pittsburgh Medical Center electronic medical record and our Head and Neck oncologic data repository. Data collected on each patient included demographics, diagnosis date, last follow-up date, primary tumor location, molecular profiles, tumor staging, and examination findings, which were compiled into the data base. We included all patients with a diagnosis of HNSCC who underwent definitive surgery, radiation, chemotherapy, or combined therapeutic modalities and had posttreatment PET/CTs performed between 2006 and 2018. Patients who had non-squamous cell malignancies or inadequate clinical or imaging data were excluded. Pretreatment tumor staging was performed on the basis of the American Joint Committee on Cancer (AJCC) AJCC Cancer Staging Handbook, 7 th edition. ${ }^{11}$

The NI-RADS categories are independently applied to the primary tumor bed $(\mathrm{P})$, the cervical lymph nodes $(\mathrm{N})$, and distant disease (D) so that every examination has 3 NI-RADS values assigned to it. The overall NI-RADS score is considered to be the maximum of the 3 values. We selected patients whose posttreatment PET/CTs were interpreted as NI-RADS 3 (suspicious) or 4 (definitive recurrence) at $\mathrm{P}, \mathrm{N}$, or $\mathrm{D}$. The PET/CT features of NI-RADS 3 at the primary site include residual or a new discrete nodule or mass with increased enhancement and/or focal FDG avidity. The features of NI-RADS 3 at the nodal sites include a progressively enlarging softtissue mass or lymph node that is worrisome for residual or recurrent tumor. NI-RADS 4 examinations include patients with definite PET/CT evidence of disease progression such as definitive locoregional recurrence or unequivocal metastases in distant organs.

\section{PET/CT Parameters}

PET/CT scans were completed by using 1 of several clinical scanners (Discovery; GE Healthcare; and Somatom Emotion; Siemens). Patients were instructed to fast at least 4-6 hours before the examination and were required to have a blood glucose level measuring $<200 \mathrm{mg} / \mathrm{dL}$ preceding the scan. Patients who did not meet these criteria were rescheduled. Patients were injected with $10-20 \mathrm{mCi}$ of $\left[{ }^{18} \mathrm{~F}\right] \mathrm{FDG} 60$ minutes before obtaining PET emission images. Immediately before PET acquisition, contrast-enhanced $(125 \mathrm{~mL}$ iopamidol, Isovue-370; Bracco) helical CT (pitch $=1.5-2.0 ; \mathrm{kV}$ (peak) $=120-140$; variable mAs; 3.75-mm collimation) was performed approximately 45 seconds after contrast injection. Images were obtained from the top of the skull through the upper thighs, with PET and CT scans matched and optimized for visualization with CT attenuation-corrected reconstruction. Patients were imaged with arms at their sides. The images of the thorax, abdomen, and pelvis were reconstructed in a $3.75-\mathrm{mm}$ section thickness with a full-body FOV. The images of the head and neck part were reconstructed in a $2.5-\mathrm{mm}$ section thickness with a small FOV. An additional high-resolution chest CT was performed with arms raised to better assess the lung parenchyma.

\section{Image Interpretation}

All PET/CT surveillance studies were interpreted by board-certified neuroradiologists within a dedicated head and neck imaging practice. Postprocessing fusion software (Mirada; Mirada Medical, Denver, Colorado) was used to assist in interpretation. Categorization was based on the subjective interpretation of the interpreting radiologist; standard uptake value thresholds were not used.

\section{Treatment and Surveillance Protocols}

Patient treatment protocols, including radiation dose and chemotherapy regimen, were determined by the standard practice guidelines of the multidisciplinary head and neck oncology team at our institution. Clinical follow-up was performed at 2-month intervals. The first surveillance PET/CT was performed 2-3 months after the completion of therapy according to established institutional protocols. Subsequent radiologic examinations were performed at 3month intervals after the first surveillance scan.,10 Additional PET/CT and tissue biopsy were performed outside the standard surveillance protocol if patients had any clinical signs and symptoms or radiographic findings suspicious for residual or recurrent tumor. The reference standard for treatment failure was confirmation by histopathology or unequivocal evidence of disease progression on subsequent follow-up imaging and clinical evaluation.

\section{Statistical Methods}

Positive predictive value was calculated separately for NI-RADS category 3 and NI-RADS category 4 and was further broken down by primary, nodal, and distant disease. PPV was calculated as the probability of experiencing treatment failure given a NIRADS category 3 or 4 . Ninety-five percent confidence intervals for percentages were calculated using binomial methods, except when the calculated value was $100 \%$, in which case exact methods were used.

\section{RESULTS}

In our complete data base, a total of 8768 PET/CT examinations were performed on 3853 patients with head and neck cancer between 2006 and 2018. Of these, 197 patients met the inclusion criteria, with 128 patients classified as NI-RADS 3 and 69 patients classified as NI-RADS 4. Most patients were male ( $n=148,75 \%)$ with an oropharyngeal primary tumor site $(n=76,39 \%)$ and stage IV disease $(n=149,76 \%)$. Forty-four of 76 patients with an oropharyngeal tumor were positive for human papillomavirus (HPV) (58\%). Patient demographics, tumor characteristics, and Tumor, Node, Metastasis staging of NI-RADS 3 and 4 are summarized in Table 1.

Seventy-two of 128 patients with posttreatment PET/CT interpreted as NI-RADS 3 at $\mathrm{P}, \mathrm{N}$, or D were proved to have treatment 


\begin{tabular}{|c|c|c|}
\hline & NI-RADS $3(n=128)$ & NI-RADS $4(n=69)$ \\
\hline \multicolumn{3}{|l|}{ Sex } \\
\hline Male & 97 (76\%) & 51 (74\%) \\
\hline Female & $31(24 \%)$ & $18(26 \%)$ \\
\hline Age (yr) & $\begin{array}{c}\text { Range }=27-87, \text { mean }=59, \\
\text { median }=60\end{array}$ & $\begin{aligned} \text { Range }= & 26-87, \text { mean }=62, \\
& \text { median }=64\end{aligned}$ \\
\hline \multicolumn{3}{|l|}{ Primary tumor location } \\
\hline Oropharynx & $55(43 \%)$ & $21(30 \%)$ \\
\hline Oral cavity & $32(25 \%)$ & $26(38 \%)$ \\
\hline Larynx & $29(23 \%)$ & $14(20 \%)$ \\
\hline Hypopharynx & $5(4 \%)$ & $4(6 \%)$ \\
\hline $\begin{array}{l}\text { Paranasal sinuses/nasal } \\
\text { cavity }\end{array}$ & $3(2 \%)$ & $3(4 \%)$ \\
\hline Nasopharynx & $2(1.5 \%)$ & $1(2 \%)$ \\
\hline Unknown & $2(1.5 \%)$ & $0(0 \%)$ \\
\hline \multicolumn{3}{|l|}{ HPV status (oropharynx) } \\
\hline Positive & $32(58 \%)$ & $12(57 \%)$ \\
\hline Negative & $18(33 \%)$ & $2(10 \%)$ \\
\hline Unknown & $5(9 \%)$ & $7(33 \%)$ \\
\hline \multicolumn{3}{|l|}{ TNM stage (7th ed $A J C C^{11}$ ) } \\
\hline Stage I & $3(2 \%)$ & $4(6 \%)$ \\
\hline Stage II & $12(10 \%)$ & $2(3 \%)$ \\
\hline Stage III & $20(16 \%)$ & $5(7 \%)$ \\
\hline Stage IV & $91(71 \%)$ & $58(84 \%)$ \\
\hline Unknown primary & $2(1 \%)$ & $0(0 \%)$ \\
\hline
\end{tabular}

Note:-TNM indicates Tumor, Node, Metastasis.

$\begin{aligned} & \text { Table 2: Timing of posttreatment PET/CT with false-positive } \\
& \text { NI-RADS } 3 \text { results }(\boldsymbol{n}=\mathbf{5 6})\end{aligned}$
\begin{tabular}{cc} 
Time Interval between Completion & No. of Posttreatment \\
of Therapy and Posttreatment PET/ & PET/CTs with False- \\
CT with False-Positive NI-RADS 3 & Positive NI-RADS 3 \\
Results & Results \\
\hline $0-3 \mathrm{mo}$ & $27(48 \%)$ \\
$3-6 \mathrm{mo}$ & $10(18 \%)$ \\
$6-12 \mathrm{mo}$ & $10(18 \%)$ \\
$1-2 \mathrm{yr}$ & $6(11 \%)$ \\
$2-3 \mathrm{yr}$ & $0(0 \%)$ \\
$3-4 \mathrm{yr}$ & $1(2 \%)$ \\
$4-5 \mathrm{yr}$ & $2(3 \%)$ \\
\hline
\end{tabular}

failure at the suspicious sites, yielding an overall PPV of 56\% (95\% CI, $48 \%-65 \%)$. The PPVs of NI-RADS 3 by subsite were as follows: P, 56\% (44/79); N, 65\% (34/52); and D, 79\% (42/53). (The denominators do not add up to 128 because some patients were classified as NI-RADS 3 at multiple subsites.) The median time interval between completion of therapy and obtaining PET/CT with NIRADS 3 was 4 months (range, 2-85 months). Most posttreatment PET/CT with false-positive NI-RADS 3 results was performed within the first 3 months after conclusion of therapy (27/56, 48\%; range, $1.5-58$ months; median, 3 months). The timing of posttreatment PET/CT with false-positive NI-RADS 3 results is summarized in Table 2.

Thirty-one of 56 patients (55\%) with false-positive NI-RADS 3 findings had histopathologic confirmation; 25 patients had no tissue confirmation but had clinical and radiologic follow-up, which demonstrated interval resolution of suspicious findings. With pathologic confirmation, the major causes of false-positives are treatment-related changes $(12 / 31,39 \%)$ and nonspecific infectious/inflammatory processes $(10 / 31,32 \%)$. Five patients with false-positive NI-RADS 3 findings had incidental benign head and neck tumor on surveillance PET/CT (Warthin tumors $=3$, pleomorphic adenoma $=$ 1 , dermatofibroma $=1$ ). Most patients with false-positive NI-RADS 3 findings had oropharyngeal squamous cell carcinoma $(24 / 56,43 \%)$, with most of these positive for HPV (15/24, 63\%). The PPVs of NI-RADS 3 in HPVrelated and HPV-unrelated oropharyngeal cancers are not substantially different (HPV-related oropharyngeal cancers: $n=32, \mathrm{PPV}=53 \%$ [17/32]; HPV-unrelated oropharyngeal cancers: $n=18, \mathrm{PPV}=56 \%[10 / 18])$.

All 69 patients with posttreatment PET/CT interpreted as NI-RADS 4 had true treatment failure, yielding a PPV of $100 \%$ (95\% CI, 96\%-100\%). Broken down by subsite, these percentages are the following: $\mathrm{P}, 100 \%(28 / 28) ; \mathrm{N}$, $100 \%(32 / 32)$; and D, 100\% (29/29). Thirty-eight of 69 patients (55\%) with NI-RADS 4 were confirmed with histopathology; 31 patients had no tissue confirmation but had unequivocal treatment failure based on follow-up imaging and clinical examination. The median time interval between completion of therapy and obtaining PET/CT with NI-RADS 4 was 3.4 months (range, 1-158 months).

Representative PET/CT images of the patients with trueand false-positive NI-RADS 3 findings are shown in Figs 1 and 2, respectively. Representative PET/CT images of a patient with NIRADS 4 are shown in Fig 3.

\section{DISCUSSION}

This study demonstrates a very high PPV (100\%) of NI-RADS 4 in posttreatment PET/CT surveillance of HNSCC. With appropriate standardization of imaging and reporting practices, this could potentially obviate biopsy confirmation. In contrast, the PPV of NI-RADS 3 in posttreatment PET/CT is relatively low, especially at the primary tumor site. Therefore, patients with "high-suspicion" PET/CT findings should be further investigated with tissue sampling before instituting new treatment regimens to avoid unnecessary overtreatment and its associated toxicities. The results of our study support the current American College of Radiology recommendations for NI-RADS 3 and 4 as well as our recommendations for posttreatment $\mathrm{PET} / \mathrm{CT}$ surveillance in HNSCC., ${ }^{9,10}$ The University of Pittsburgh PET/CT surveillance algorithm for patients with HNSCC is shown in Fig 4. The ultimate goal of the surveillance protocol is to identify recurrences to potentially treat and cure, while maximizing cost-efficiency without increased morbidity and mortality.

The PPV of NI-RADS 3 in our study is similar to that in the previous study of the initial performance of NI-RADS. ${ }^{12}$ Krieger et $\mathrm{al}^{12}$ achieved an overall PPV of NI-RADS 3 of $59.4 \%$ (19/32), with a PPV of $54.6 \%$ at the primary tumor site $(12 / 22)$ and a PPV of $70 \%$ 

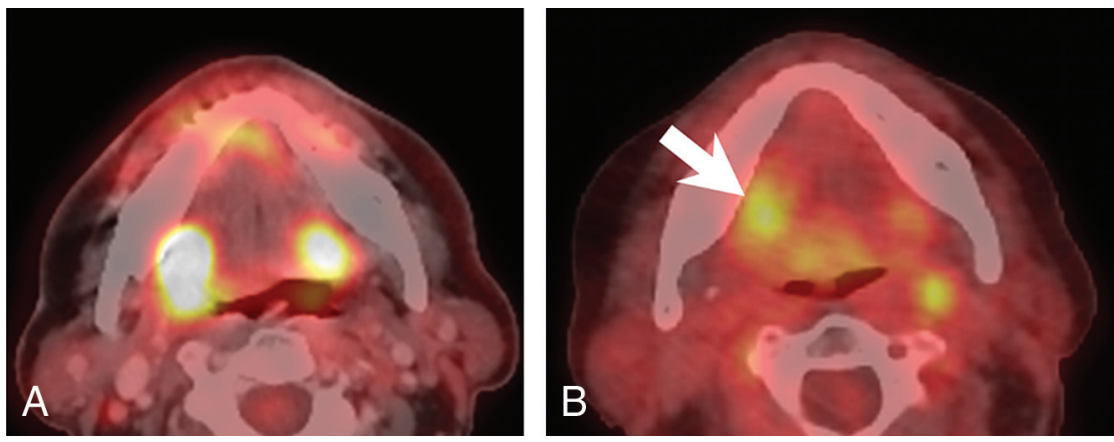

FIG 1. NI-RADS 3 true-positive. A 62-year-old man with advanced HPV-positive oropharyngeal squamous cell carcinoma. A, Pretreatment PET/CT shows 2 areas of FDG-avid infiltrative tumor in the oropharynx, one centered in the right faucial tonsil with invasion of the adjacent right tongue base and right-sided floor of mouth and one centered in the left tongue base. $B$, Surveillance PET/CT obtained at 3.5 months after completion of treatment shows interval improvement and a decrease in the size of the primary tumor, but there remains a substantial amount of residual FDG avidity in the right-sided floor of the mouth (white arrow), which is suspicious for residual viable tumor. Treatment failure was subsequently confirmed with biopsy.
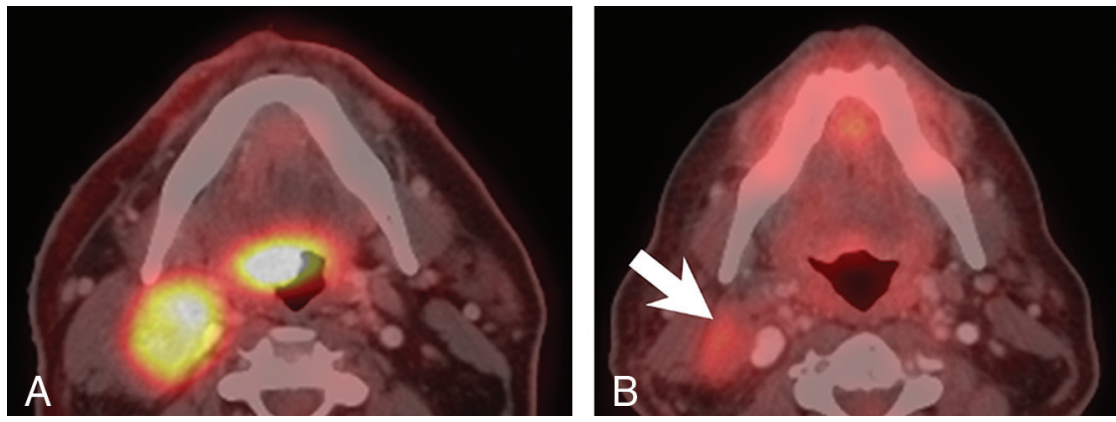

FIG 2. NI-RADS 3 false-positive. A 55-year-old man with metastatic HPV-positive oropharyngeal squamous cell carcinoma. A, Pretreatment PET/CT shows a large FDG-avid right oropharyngeal tumor with FDG-avid right level lla nodal metastasis. B, Surveillance PET/CT obtained at 2.5 months after completion of treatment shows complete response of the primary tumor, but there remains a large nodal remnant with moderate FDG-avidity (white arrow), which is concerning for residual viable tumor. The patient subsequently underwent right-neck dissection, with final pathology showing treatment-related changes but no viable tumor.
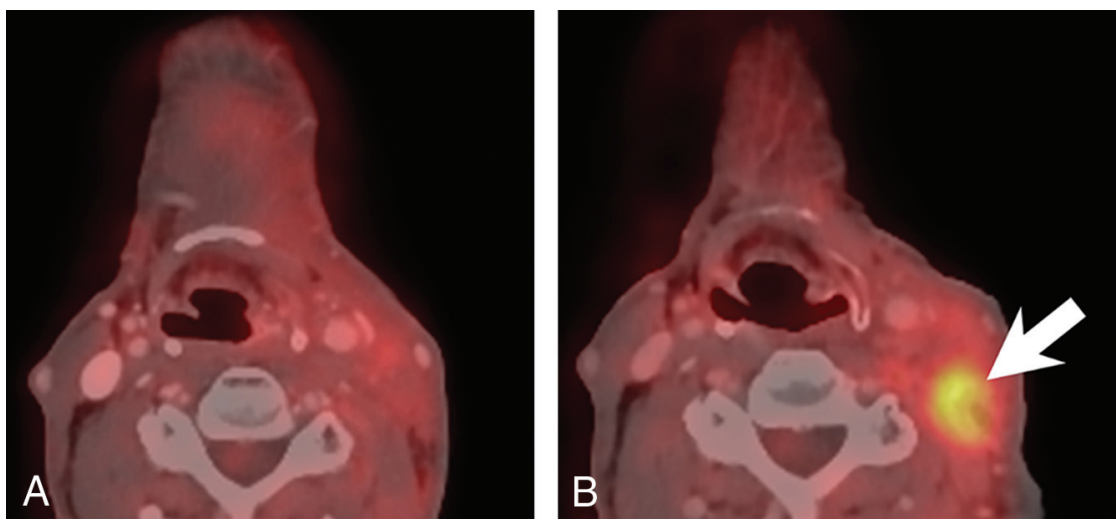

FIG 3. NI-RADS 4 true-positive. A 74-year-old man with squamous cell carcinoma of the oral cavity after tumor resection, left-neck dissection, and adjuvant chemoradiation. $A$, The first surveillance PET/CT obtained at 3.5 months after completion of treatment shows treatment-related changes, with no convincing imaging evidence of viable tumor. $B$, At 8 months after completion of treatment, there is a new FDG-avid soft-tissue mass in the left upper neck (white arrow), indicative of locoregional tumor recurrence. at the nodal site (7/10). Similarly, the PPV for the NI-RADS 3 in primary tumors of our study was also lower than for the lymph nodes (56\% versus $65 \%$ ). A previous study of posttreatment PET/CT in oropharyngeal squamous cell carcinoma likewise demonstrated a low PPV for PET/CT findings of residual FDG uptake suspicious for locoregional recurrence $(n=30$, overall $\mathrm{PPV}=40 \%$, PPV for primary tumor site $=20 \%$, PPV for nodal site $=29 \%) .{ }^{13}$ As expected, treatmentrelated changes and superimposed infection are the major causes of falsepositive NI-RADS 3 results, which account for $71 \%$ of patients who had histopathologic confirmation. The primary reason for low PPVs in NI-RADS 3 is likely due to a significant overlap of PET/CT features between viable tumors and inflammatory changes. This may also explain why NI-RADS 3 on posttreatment PET/CT has a slightly higher false-positive rate compared with contrast-enhanced CT alone. ${ }^{12}$

Another cause of false-positive NIRADS 3 findings is incidental FDGavid head and neck neoplasms. In our study, 5 patients were found to have incidental benign head and neck neoplasms, with a substantial proportion from major salivary gland tumors. Warthin tumor is one known head and neck tumor that has variable FDG uptakes, one of the pitfalls in the interpretation of head and neck PET/ CT. ${ }^{14-16}$ These incidental neoplasms can mimic metastases and have an impact on the management of patients with HNSCC. Care should be taken when interpreting posttreatment head and neck PET/CT, with careful evaluation of the contrast-enhanced CT portion. Tissue sampling may also be warranted and used as a problemsolving tool in some cases.

The AJCC Cancer Staging Handbook, 8th edition, has classified oropharyngeal cancers into 2 different subtypes based on the HPV profile (p16 overexpression) due to their differences in natural history and prognosis. HPV-related oropharyngeal cancers more commonly occur in younger and healthier individuals, with no history of significant exposure to tobacco, and have better 


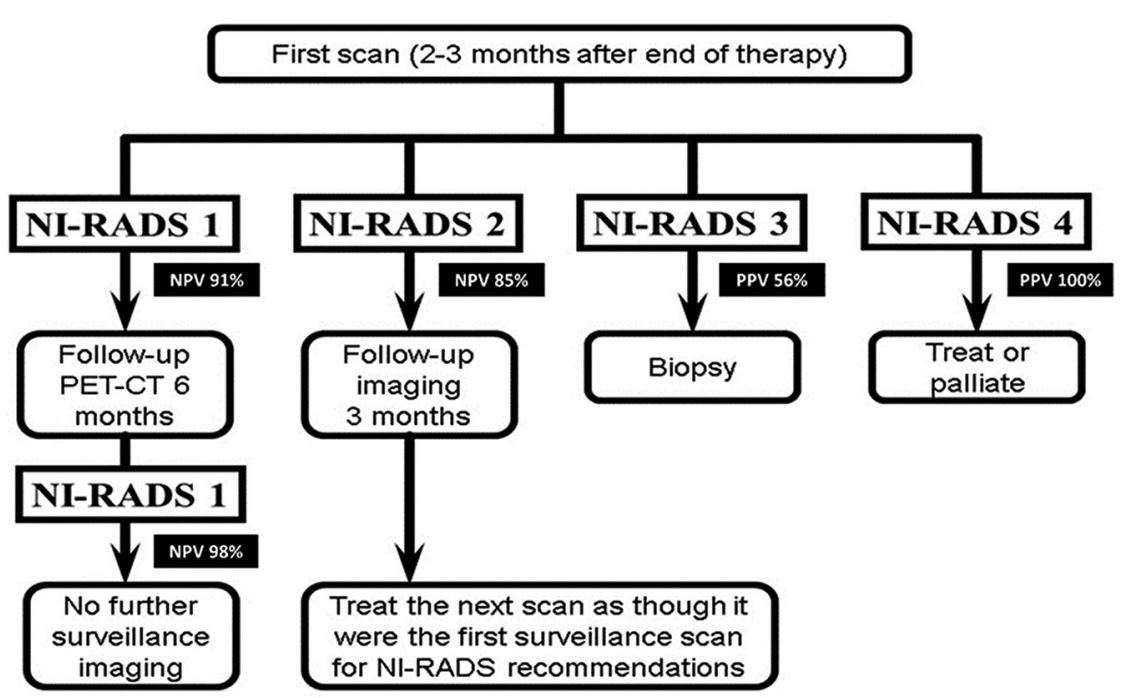

FIG 4. University of Pittsburgh PET/CT Surveillance Flowchart for Head and Neck Squamous Cell Carcinoma. NI-RADS 1, no evidence of recurrence; NI-RADS 2, low suspicion; NI-RADS 3, high suspicion; NI-RADS 4, definitive disease recurrence. Negative predictive values of first NI-RADS 1 $\mathrm{PET} / \mathrm{CT}=91 \%$; negative predictive values of 2 consecutive NI-RADS $1 \mathrm{PET} / \mathrm{CTs}=98 \%{ }^{5}$, negative predictive values of the first NI-RADS 2 PET/CT $=85 \%{ }^{10}$ PPV of NI-RADS 3 PET/CT $=56 \%$; PPV of NI-RADS 4 PET/CT $=100 \%$.

prognosis compared with HPV-unrelated cancers. ${ }^{17}$ Radiologically, HPV-related cancers tend to have more cystic changes in metastatic lymph nodes and distant metastases at unusual sites such as bones or brain. ${ }^{18}$ However, the impact of the HPV profile on posttreatment PET/CT results remains unknown. Based on our current data base with a small sample size, the PPVs of NI-RADS 3 in HPV-related and HPV-unrelated oropharyngeal cancers are similar (HPV-related oropharyngeal cancers: $n=32, \mathrm{PPV}=53 \%$ [17/32]; HPV-unrelated oropharyngeal cancers: $n=18$, PPV $=$ $56 \%[10 / 18])$. Further study with a larger sample size is needed for investigation of the potential impact of the new cancer staging system on posttreatment PET/CT results.

This study has several limitations. First, the study is inherently limited due to its retrospective study design. The raw data that we used for retrospective analysis were collected prospectively into a registry conducted at 1 center using institution-specific PET/CT protocols and experienced head and neck neuroradiologists. The results of the data are generated from the head and neck oncologic data repository, which is completed via chart review and dependent on the accuracy of both the repository and the patient chart. The standard uptake value was not used in PET/CT interpretation because there is no standard cutoff standard uptake value threshold that reliably differentiates benign and malignant processes. ${ }^{19,20}$ We used the AJCC Cancer Staging Handbook, 7th edition, because it was used in the management of the patients during the time of data collection. Last, we included all posttreatment PET/CTs that were obtained at any time after completion of therapy. We did not limit our analysis to the PPV of NI-RADS 3 and 4 on the first posttreatment PET/CT because of the small sample size. Thus, the results are not entirely specific to posttreatment PET/ $\mathrm{CT}$ at any 1 particular time after treatment. Our subgroup analysis of NI-RADS 3, though insufficiently powered, suggests that

\section{REFERENCES} 94 CrossRef Medline CrossRef Medline Medline 46 CrossRef Medline most false-positive NI-RADS 3 findings occur within 3 months after the conclusion of treatment, though further study with a larger sample size is required.

\section{CONCLUSIONS}

In the setting of PET/CT for surveillance of treated head and neck squamous cell carcinoma, NI-RADS category 4 has a superb positive predictive value and may obviate tissue confirmation. In contrast, the positive predictive value of NI-RADS category 3 on posttreatment PET/CT is relatively low, particularly at the primary tumor site. Therefore, confirmation of NI-RADS category 3 findings should be performed with tissue sampling before instituting new treatment regimens to avoid unnecessary overtreatment and its associated toxicities.

Disclosures: Umamaheswar Duvvuri-UNRELATED: Board Membership: Activ Surgical; Consultancy: Medtronic; Grants/Grants Pending: National Institutes of Health R01-DE028343 and Veterans Administration 101BX003456; Patents (Planned, Pending or Issued): patent pending for robotic surgery instrument.* *Money paid to the institution.

1. Goel R, Moore W, Sumer B, et al. Clinical practice in PET/CT for the management of head and neck squamous cell cancer. AJR Am J Roentgenol 2017;209:289-303 CrossRef Medline

2. Tantiwongkosi B, Yu F, Kanard A, et al. Role of (18)F-FDG PET/CT in pre and post treatment evaluation in head and neck carcinoma. World J Radiol 2014;6:177-91 CrossRef Medline

3. Kale H, Rath TJ. Chapter 3: the role of PET/CT in squamous cell carcinoma of the head and neck. Semin Ultrasound CT MR 2017;38:479-

4. Mehanna H, Wong WL, McConkey CC, et al; PET-NECK Trial Management Group. PET-CT surveillance versus neck dissection in advanced head and neck cancer. N Engl J Med 2016;374:1444-54

5. McDermott M, Hughes $M$, Rath $T$, et al. Negative predictive value of surveillance PET/CT in head and neck squamous cell cancer. AJNR Am J Neuroradiol 2013;34:1632-36 CrossRef Medline

6. Nayak JV, Walvekar RR, Andrade RS, et al. Deferring planned neck dissection following chemoradiation for stage IV head and neck cancer: the utility of PET-CT. Laryngoscope 2007;117:2129-34 CrossRef

7. Aiken AH, Farley A, Baugnon KL, et al. Implementation of a novel surveillance template for head and neck cancer: Neck Imaging Reporting and Data System (NI-RADS). J Am Coll Radiol 2016;13:743-

8. Aiken AH, Hudgins PA. Neck Imaging Reporting and Data System. Magn Reson Imaging Clin N Am 2018;26:51-62 CrossRef Medline

9. Aiken AH, Rath TJ, Anzai Y, et al. ACR Neck Imaging Reporting and Data Systems (NI-RADS): a white paper of the ACR NI-RADS Committee. J Am Coll Radiol 2018;15:1097-1108 CrossRef Medline

10. Wangaryattawanich $P$, Branstetter BFt, Hughes $M$, et al. Negative predictive value of NI-RADS category 2 in the first posttreatment FDG-PET/CT in head and neck squamous cell carcinoma. AJNR Am J Neuroradiol 2018;39:1884-88 CrossRef Medline 
11. Edge SB, Byrd DR, Compton CC, et al. AJCC Cancer Staging Handbook: From the AJCC Cancer Staging Manual. 7th ed. SpringerVerlag 2010;53:39-126

12. Krieger DA, Hudgins PA, Nayak GK, et al. Initial performance of NI-RADS to predict residual or recurrent head and neck squamous cell carcinoma. AJNR Am J Neuroradiol 2017;38:1193-99 CrossRef Medline

13. Bird T, Barrington S, Thavaraj S, et al. (18)F-FDG PET/CT to assess response and guide risk-stratified follow-up after chemoradiotherapy for oropharyngeal squamous cell carcinoma. Eur J Nucl Med Mol Imaging 2016;43:1239-47 CrossRef Medline

14. Purohit BS, Ailianou A, Dulguerov N, et al. FDG-PET/CT pitfalls in oncological head and neck imaging. Insights Imaging 2014;5:585602 CrossRef Medline

15. Rassekh CH, Cost JL, Hogg JP, et al. Positron emission tomography in Warthin's tumor mimicking malignancy impacts the evaluation of head and neck patients. Am J Otolaryngol 2015;36:259-63 CrossRef Medline
16. Schwarz E, Hurlimann S, Soyka JD, et al. FDG-positive Warthin's tumors in cervical lymph nodes mimicking metastases in tongue cancer staging with PET/CT. Otolaryngol Head Neck Surg 2009; 140:134-35 CrossRef Medline

17. Huang SH, O'Sullivan B. Overview of the 8th Edition TNM Classification for Head and Neck Cancer. Curr Treat Options Oncol 2017;18:40 CrossRef Medline

18. Subramaniam RM, Alluri KC, Tahari AK, et al. PET/CT imaging and human papilloma virus-positive oropharyngeal squamous cell cancer: evolving clinical imaging paradigm. J Nucl Med 2014;55:431-38 CrossRef Medline

19. Manca G, Vanzi E, Rubello D, et al. (18)F-FDG PET/CT quantification in head and neck squamous cell cancer: principles, technical issues and clinical applications. Eur J Nucl Med Mol Imaging 2016;43:1360-75 CrossRef Medline

20. de Langen AJ, Vincent A, Velasquez LM, et al. Repeatability of $\mathbf{1 8 F}$ FDG uptake measurements in tumors: a metaanalysis. J Nucl Med 2012;53:701-08 CrossRef Medline 